\title{
VINCES UG-03 y VINCES UG-10, NUEVAS VARIEDADES DE ARROZ PARA LA COSTA ECUATORIANA
}

\author{
VINCES UG-03 AND VINCES UG-10, NEW RICE CULTIVARS \\ FOR THE ECUADORIAN COAST
}

\author{
Vicente Painii-Montero' ', Gardenia González-Manjarrez', Olimpa \\ Santillan-Muñóz y yelipe R. Garcés-Fiallos ${ }^{2,3 *}$
}

\begin{abstract}
'Facultad de Ciencias para el Desarrollo, Universidad de Guayaquil. km 1/2 vía Vinces-Palestina. EC120850, Vinces, Ecuador. ${ }^{2}$ Facultad de Ciencias Agrarias, Universidad de Guayaquil. Cdla. Universitaria "Salvador Allende. EC090302, Malecón del Salado entre Av. Delta y Av. Kennedy, Guayaquil, Guayas, Ecuador. ${ }^{3}$ Campus Experimental La Teodomira, Facultad de Ingeniería Agronómica, Universidad Técnica de Manabí. km 13 1⁄2 vía Santa Ana. EC130105, Portoviejo, Manabí, Ecuador.
\end{abstract}

*Autor para correspondencia (felipe.garcesf@ug.edu.ec; felipegarces23@yahoo.com)

El cultivo de arroz (Oriza sativa L.) es la principal fuente alimenticia en la dieta básica de los habitantes de la costa ecuatoriana (Garcés-Fiallos et al., 2012). Este cultivo está establecido principalmente en las provincias de Los Ríos y Guayas (INEC, 2016a). En los años 2014 y 2015 el rendimiento nacional promedio fue 3900 y $4410 \mathrm{~kg} \mathrm{ha}^{-1}$, respectivamente (INEC, 2016b).

En Ecuador existen variedades de arroz como INIAP-17, cuyo rendimiento puede superar los $4000 \mathrm{~kg} \mathrm{ha}^{-1}$ en condiciones experimentales óptimas de manejo (Rodríguez et al., 2017). Sin embargo, este rendimiento aún es bajo si se le compara con el obtenido en países como Brasil bajo condiciones experimentales similares, donde se obtienen $7000 \mathrm{~kg} \mathrm{ha}^{-1}$ (Santos et al., 2017).

El proceso de mejoramiento genético que se aplicó para obtener estas variedades fue el método genealógico o de pedigrí durante seis generaciones (2006 a 2012). La variedad Vinces UG-03 proviene de selecciones individuales (planta por surco) a partir de los mejores genotipos de una selección masal inicial del criollo Chapulo, colectado en la localidad Fruta de Pan, Ventanas, provincia de Los Ríos. Esta variedad proviene de la selección UG008-1P2P-4P-5P-7P, y su pedigrí es UG012-2P-4P-5P-8P-M. La variedad Vinces UG-10 proviene de la cruza entre las líneas CC-03 (progenitor femenino) y CC-05 (progenitor masculino). La línea CC-03 fue seleccionada del criollo Suástegui-1, colectado en la localidad Santa Martha; la línea CC-05 proviene del criollo Suarroz-1, colectado en la localidad Los Vergeles, ambos en la provincia de Los Ríos. Esta variedad proviene del cruce original UG005-1P3P-5P-M-3P $\times$ UG008-3P-6P-8P-M-3P y su pedigrí es UG010-1P-3P-5P-6P-8P-M.

Las variedades Vinces UG-03 y Vinces UG-10 se seleccionaron con base en su aspecto de planta, potencial productivo y resistencia a enfermedades. La superioridad de estos genotipos se confirmó en evaluaciones agronómicas, de rendimiento y de calidad culinaria (cocción utilizando un proceso convencional), durante el período 2011 a 2013, en doce ambientes de la Cuenca del Río Guayas (Cuadros 1 y 2). El rendimiento promedio de las variedades Vinces UG-03 y Vinces UG-10 (6388 $\left.\mathrm{kg} \mathrm{ha}^{-1}\right)$ fue $15 \%$ mayor que el de las variedades testigo (5413 $\mathrm{kg} \mathrm{ha}^{-1}$ ) (Cuadro 1).

Vinces UG-03 y Vinces UG-10, poseen excelentes características agronómicas, pues con base en los criterios de IRRI (2013) y de Araújo et al. (2011) para Diatraea saccharalis, ambas variedades son resistentes a factores bióticos como Rice Hoja Blanca Virus (RHBV), tizón de la vaina (Rhizoctonia spp.), falso carbón (Ustilaginoidea virens) y barrenador (Diatraea saccharalis), así como tolerantes a la quemazón (Pyricularia grisea), mancha marrón (Bipolaris sp.), pudrición de la vaina (Sarocladium oryzae), pudrición negra del pie (Gaeumannomyces graminis var. graminis), manchado del grano (varios patógenos fúngicos); además, cuentan con excelente calidad molinera y culinaria.

Con relación con la calidad de grano (Cuadro 2), las variedades VINCES UG-03 y VINCES UG-10 presentan buen tamaño de grano (entre grano largo y extra largo) y un índice promedio de pilado de $62.2 \%$. Las pruebas de cocción mostraron que el grano está entre moderadamente pegajoso a moderadamente separado. Estas características son ideales para el consumo del mercado local y su entorno.

Los genotipos se registraron el 25 de mayo de 2014 como cultivares en el Libro de Registro de Cultivares, en la Dirección de Agrobiodiversidad y Cambio Climático del Consejo Nacional de Semillas del Ecuador, con los números IX - 25 (VINCES UG-03) y IX - 26 (VINCES UG-10), conforme 
Cuadro 1. Rendimiento promedio de grano $\left(\mathrm{kg} \mathrm{ha}^{-1}\right)$ de las variedades VINCES UG-03 y VINCES UG-10, en 12 ambientes de validación entre los años 2011 y 2013.

\begin{tabular}{|c|c|c|c|c|c|c|c|c|}
\hline \multirow[b]{2}{*}{ Genotipos } & \multicolumn{3}{|c|}{ Año 2011 (época lluviosa y seca) } & \multicolumn{5}{|c|}{ Año 2012 (época seca) } \\
\hline & $\begin{array}{l}\text { Vinces } \\
\text { (secano) }\end{array}$ & $\begin{array}{l}\text { Pueblo viejo } \\
\text { (secano) }\end{array}$ & $\begin{array}{l}\text { Vinces } \\
\text { (poza) }\end{array}$ & $\begin{array}{l}\text { Vinces } \\
\text { (secano) }\end{array}$ & $\begin{array}{l}\text { Vinces } \\
\text { (poza) }\end{array}$ & $\begin{array}{c}\text { Baba } \\
\text { (piscina) }\end{array}$ & $\begin{array}{c}\text { Santa Lucía } \\
\text { (piscina) }\end{array}$ & $\begin{array}{l}\text { Palestina } \\
\text { (piscina) }\end{array}$ \\
\hline VINCES UG-03 & $6052 b^{1}$ & $4356 \mathrm{~b}$ & 8769 a & 5847 a & $8575 b$ & 5988 bc & $7278 b$ & 6100 a \\
\hline VINCES UG-10 & $7001 \mathrm{c}$ & $5113 \mathrm{c}$ & 8411 a & 4851 a & $8108 b$ & $6228 c$ & $7083 a b$ & 5252 a \\
\hline INIAP-14 & 4821 a & $2121 \mathrm{a}$ & 8262 a & 4821 a & $8605 b$ & $5572 b$ & $7497 b$ & 4715 a \\
\hline INIAP-16 & 4876 a & 1742 a & 8675 a & 4876 a & 6973 a & 4924 a & 6194 a & 5504 a \\
\hline Promedio & 5688 & 3333 & 8529 & 5099 & 8065 & 5678 & 7013 & 5393 \\
\hline \multirow[t]{3}{*}{ CV (\%) } & 7 & 10 & 8 & 13 & 5 & 4 & 6 & 12 \\
\hline & \multicolumn{4}{|c|}{ Año 2013 (época lluviosa) } & & & & \\
\hline & $\begin{array}{l}\text { Vinces } \\
\text { (secano) }\end{array}$ & $\begin{array}{l}\text { Colimes } \\
\text { (piscina) }\end{array}$ & $\begin{array}{c}\text { Santa } \\
\text { Lucía } \\
\text { (piscina) }\end{array}$ & $\begin{array}{c}\text { Puebloviejo } \\
\text { (secano) }\end{array}$ & Promedio & & & \\
\hline VINCES UG-03 & $5557 \mathrm{~b}$ & $7075 b$ & 6596 a & $4358 \mathrm{~b}$ & $6379 b$ & & & \\
\hline VINCES UG-10 & $6485 b$ & $6858 b$ & $6900 \mathrm{~b}$ & $4481 b$ & $6398 b$ & & & \\
\hline INIAP-14 & 2985 a & $6881 b$ & $5588 \mathrm{ab}$ & 2922 a & 5399 a & & & \\
\hline INIAP-16 & $6099 b$ & 6229 a & 5082 a & $3966 a b$ & 5428 a & & & \\
\hline Promedio & 5282 & 6761 & 6042 & 3932 & & & & \\
\hline CV $(\%)$ & 10 & 2 & 10 & 13 & & & & \\
\hline
\end{tabular}

${ }^{\top}$ Medias seguidas por la misma letra en la misma columna no difieren significativamente (Tukey, P $\left.\leq 0.05\right)$.

a la normativa ecuatoriana dentro de la Ley de Semillas vigente, Capítulo II del Registro de Cultivares, Artículos 2 al 8.

\section{BIBLIOGRAFÍA}

Araújo, L. F. de, A. G. da Silva, I. Cruz, E. L. do Carmo, A. H. Neto, M. M. P. Goulart e J. F. Rattes (2011) Flutuação populacional de Spodoptera frugiperda (J. E. Smith), Diatraea saccharalis (Fabricius) e Doru luteipes (Scudder) em milho convencional e transgênico Bt. Revista Brasileira de Milho e Sorgo 10:205-214, http://dx.doi. org/10.18512/1980-6477/rbms.v10n3p205-214.

Garcés-Fiallos, F. R., T. G. Díaz C. y A. J. Aguirre-C. (2012) Severidad de la quemazón (Pyricularia oryzae Cav.) en germoplasma de arroz F1 en la zona central del litoral ecuatoriano. Ciencia y Tecnología 5:1-6, https://doi.org/10.18779/cyt.v5i2.81

INEC, Instituto Nacional de Estadística y Censos (2016a) Encuesta de superficie y producción agropecuaria continua ESPAC 2016. Instituto Nacional de Estadística y Censos. Quito, Ecuador. 22 p. http://www.ecuadorencifras.gob.ec/documentos/web-inec/
Estadisticas_agropecuarias/espac/espac-2016/Informe\%20 ejecutivo\%20ESPAC_2016.pdf (Febrero 2017).

INEC, Instituto Nacional de Estadística y Censos (2016b) Encuesta de superficie y producción agropecuaria continua ESPAC 2015. Instituto Nacional de Estadística y Censos. Quito, Ecuador. 62 p. http://www.ecuadorencifras.gob.ec//documentos/web-inec/ Estadisticas_agropecuarias/espac/espac_2014-2015/2015/ Presentacion\%20de\%20resultados\%20ESPAC_2015.pdf (Marzo 2017).

IRRI, International Rice Research Institute (2013) Standard Evaluation System for Rice (SES). $5^{\text {th }}$ edition. International Rice Research Institute. Manila, Philippines. $55 \mathrm{p}$

Rodríguez G. J., E. Colina N., C. Castro A., G. García V., M. Uvidia V. y D. Santana A. (2017) Eficiencia agronómica del arroz INIAP-17 con niveles de fertilización química y biológica en el Litoral Ecuatoriano. Revista de Ciencia e Investigación 2:10-15.

Santos, A. B. dos, L. F. Stone, A. B. Heinemann e T. P. B. Santos (2017) Índices fisiológicos do arroz irrigado afetados pela inundação e fertilização nitrogenada. Revista Ceres 64:122-131, http://dx.doi. org/10.1590/0034-737×201764020003. 
Cuadro 2. Principales características de las variedades VINCES UG-03 y VINCES UG-10 de arroz, validadas en ocho ambientes entre los años 2011 y 2013.

\begin{tabular}{|c|c|c|}
\hline \multirow{2}{*}{ Variables } & \multicolumn{2}{|c|}{ Valores y/o calificación } \\
\hline & Vinces UG-03 & Vinces UG-10 \\
\hline Ciclo vegetativo (días) & 133 а 141 & 133 a 141 \\
\hline Altura de planta $(\mathrm{cm})$ & 99 a 117 & 95 a 118 \\
\hline Acame de las plantas & Resistente & Resistente \\
\hline Senescencia & Intermedia & Intermedia \\
\hline Número de panículas por planta ${ }^{\dagger}$ & 13 a 21 & 13 a 21 \\
\hline Longitud de panícula (cm) & 25 a 27 & 25 a 27 \\
\hline Fertilidad espiguilla (\%) & 83 & 82 \\
\hline Granos por panícula & 155 a 175 & 160 a 179 \\
\hline Longitud de grano $(\mathrm{mm})^{+\dagger}$ & 7.6 & 7.4 \\
\hline Forma del grano & Alargado & Alargado \\
\hline Peso de 1000 granos (g) & 27 a 30 & 27 a 30 \\
\hline Desgrane & Resistente & Resistente \\
\hline Índice de pilada (\%) & 61 & 63 \\
\hline Separación de granos después de cocción & Moderadamente pegajoso & Moderadamente pegajoso \\
\hline Centro blanco & Pequeño & Pequeño \\
\hline Rice Hoja Blanca Virus & Resistente & Resistente \\
\hline Pyricularia grisea & Tolerante & Tolerante \\
\hline Rhizoctonia spp. & Resistente & Resistente \\
\hline Ustilaginoidea virens & Resistente & Resistente \\
\hline Bipolari ssp. & Tolerante & Tolerante \\
\hline Sarocladium orizae & Tolerante & Tolerante \\
\hline Gaeumannomyces graminis & Tolerante & Tolerante \\
\hline Manchado del grano & Tolerante & Tolerante \\
\hline Diatraea saccharalis & Resistente & Resistente \\
\hline
\end{tabular}

${ }^{\dagger} 16$ plantas por $\mathrm{m}^{2} .{ }^{+\dagger} \mathrm{Grano}$ extra largo (EL) más de $7.5 \mathrm{~mm}$. 